OPEN ACCESS

Edited by:

Marcos Vinicius Calfat Maldaun,

Hospital Sírio-Libanês, Brazil

Reviewed by:

Paulo Henrique Pires De Aguiar, Catholic University of Sao Paulo,

Brazil

David D. Eisenstat,

University of Alberta, Canada

*Correspondence:

Yinhong Liu

liuyinhong2777@bjhmoh.cn

Specialty section:

This article was submitted to

Neuro-Oncology and

Neurosurgical Oncology,

a section of the journal

Frontiers in Oncology

Received: 14 February 2017

Accepted: 19 September 2017

Published: 09 October 2017

Citation:

Jiang Y, Chen J, He J, Pei A, Zhang J and Liu Y (2017) Parenchymal

Infiltration in Primary Diffuse Leptomeningeal Gliomatosis:

Dynamic Changes in Brain MRI.

Front. Oncol. 7:240.

doi: 10.3389/fonc.2017.00240

\section{Parenchymal Infiltration in Primary Diffuse Leptomeningeal Gliomatosis: Dynamic Changes in Brain MRI}

\author{
Yun Jiang ${ }^{1}$, Juan Chen², Jing $\mathrm{He}^{1}$, Ao Pei ${ }^{3}$, Jinsong Zhang ${ }^{4}$ and Yinhong Liu ${ }^{1 *}$ \\ 'Department of Neurology, Beijing Hospital, National Center of Gerontology, Beijing, China, ${ }^{2}$ Department of Radiology, \\ Beijing Hospital, National Center of Gerontology, Beijing, China, ${ }^{3}$ Department of Neurosurgery, Beijing Hospital, National \\ Center of Gerontology, Beijing, China, ${ }^{4}$ Department of Pathology, Beijing Hospital, National Center of Gerontology, \\ Beijing, China
}

Primary diffuse leptomeningeal gliomatosis (PDLG) is a rare and fatal disease with no special clinical manifestations. Here, we report the dynamic brain magnetic resonance imaging (MRI) changes in a 30-year-old female PDLG patient over a 10-month period. MRI showed aggressive dilation of the subarachnoid space and the ventricular system, numerous encapsulated cysts in the subarachnoid space and the dilated cerebral sulci, diffuse reticulated or focal nodular enhancement in the subarachnoid space, as well as overall enhancement in the cystic walls. In addition to the aforementioned PDLG pathological findings, MRI also revealed non-contrasted solid lesions and a contrasted cyst-like lesion in the paraventricular areas. The dynamic and multiform neuroradiological changes help us to understand the pathological process of PDLG. Of particular interest is the discovery that parenchymal infiltration can occur in PDLG.

Keywords: primary leptomeningeal gliomatosis, parenchyma, infiltration, brain magnetic resonance imaging, dynamics

\section{INTRODUCTION}

Primary diffuse leptomeningeal gliomatosis (PDLG) is associated with rapid disease progression and mortality. PDLG arises from heterotopic neuroglia tissue in the leptomeninges and spreads widely throughout the subarachnoid space (1). The majority of these neoplasms are often high grade astrocytic (2) and few are oligodendrogliomatosis (3). Compared with oligodendrogliomatosis $(3,4)$, the astrocyte-derived PDLG is more aggressive. Due to the non-specific and variable clinical presentation of PDLG, diagnosis relies on biopsy and histologic confirmation. As a result, most cases were only diagnosed at a late stage or postmortem. Traditionally, PDLG is confined to the leptomeninges without involvement of the brain or the spinal cord (1). Here, we report serial magnetic resonance imaging (MRI) findings from a PDLG patient over a 10-month period. In addition to dilation of the subarachnoid space and encapsulated cysts in the subarachnoid space and the dilated cortical sulci, as previously reported by other authors, the MRIs of this patient showed solid or cyst-like parenchymal infiltration. Dynamic alterations in the brain MRI findings over time help elucidate the natural history of PDLG.

\section{BACKGROUND}

A 30-year-old, previously healthy female, was first admitted to a local hospital on June 24, 2013 with main complaints of progressive headache, nausea, vomiting, and blurred vision which had persisted 

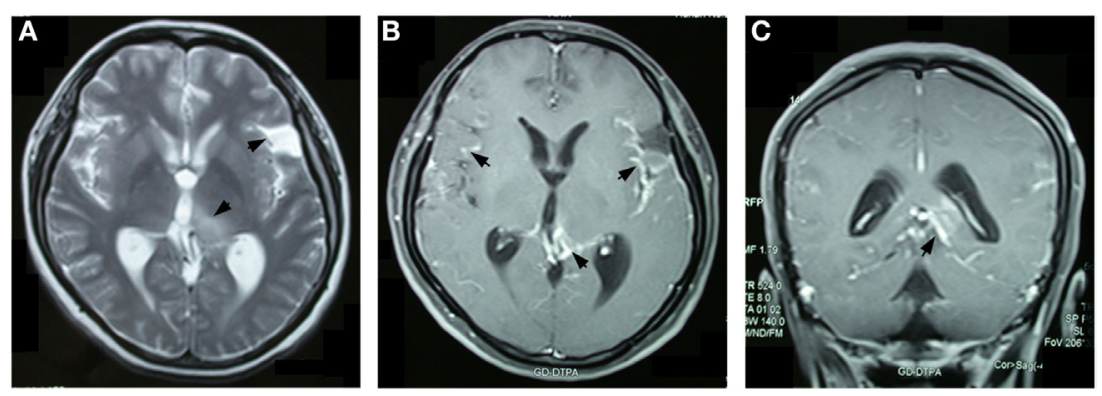

FIGURE 1 | Brain magnetic resonance imaging 2 months after the onset of symptoms revealed an abnormal signal with hyper-density on T2-weighted images in the left thalamus and the dilated left Sylvian fissure (A). Overall enhancement was present in the cerebral meningeal, the cerebellar tentorium, the pia mater around left the thalamus, and the quadrigeminal cistern, but not in the left thalamus $(\mathbf{B}, \mathbf{C})$.
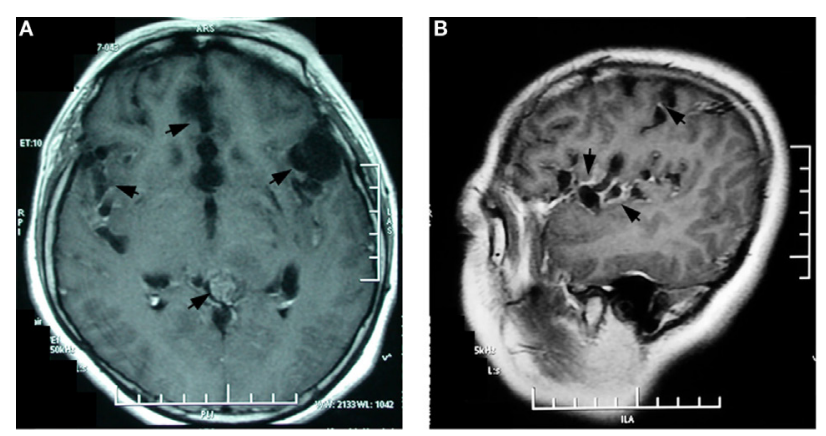

FIGURE 2 | Contrasted brain magnetic resonance imaging 9 months after symptoms onset showed multiple enhanced encapsulated cyst-like lesions in the Sylvian fissure, dilated cortex sulci in the bilateral hippocampus and the medialis of bilateral frontal lobes, and a generally enhanced mass in the quadrigeminal cistern (A). The sagittal image clearly shows grape-line cysts along the Sylvian fissure (B)

for 2 months. On admission, she was afebrile and general physical examination revealed no abnormalities. Her neurological abnormalities included bilateral papilledema, vision impairment, and signs of meningeal irritation. No motor or sensory deficits were seen. Blood tests of routine, biochemistry, coagulation, thyroid function, and various immunological parameters were all in the normal range. Blood antibodies for HIV, syphilis, and tuberculosis were negative. Initial brain MRI revealed a dilated left Sylvian fissure and an abnormal signal on the medial-dorsal side of the left thalamus (Figure 1A). Diffuse enhancement was noticed in the meninges, the left thalamus adjacent ventricular ependyma, the cerebellar tentorium, and the quadrigeminal cistern, whereas no enhancement was identified in the parenchyma (Figures 1B,C). Cerebrospinal fluid (CSF) analysis in the 3 -series lumbar punctures revealed opening pressures higher than $400 \mathrm{mmH}_{2} \mathrm{O}$ (reference range $80-180 \mathrm{mmH}_{2} \mathrm{O}$ ), with protein $2.59-5.01 \mathrm{~g} / \mathrm{L}$ (reference range $0.15-0.45 \mathrm{~g} / \mathrm{L}$ ), glucose 2.20-2.79 $\mathrm{mmol} / \mathrm{L}$ (reference range $2.5-4.5 \mathrm{mmol} / \mathrm{L}$ ), chloride 121.1-126.3 $\mathrm{mmol} / \mathrm{L}$ (reference range $120-130 \mathrm{mmol} / \mathrm{L}$ ), and WBC $3-6 / \mathrm{mL}$ (reference range $0-5 / \mathrm{mL}$ ) with $80-85 \%$ of lymphocytes. Negative results were reported in the following CSF etiological investigations: polymerase chain reaction for tuberculosis, bacteria and fungi cultures, India Ink stain for Cryptococcus, and IgG and IgM antibodies targeting various viruses and parasites. CSF cytology studies did not reveal neoplasm and chest CT and abdomen CT scans did not indicate any abnormality. Tuberculous meningitis was suspected and treated with isoniazid, rifampicin, ethambutol, and pyrazinamide. Meanwhile, brain ventricular puncture and drainage were performed and this dramatically attenuated the headache symptoms. The extraventricular drainage device was removed after 2 weeks and shortly thereafter the patient suffered from generalized tonic-clonic seizures. Once the seizures were controlled, a ventricular-peritoneal shunt was placed, resulting in an improvement of the patient's symptoms. Anti-tubercular therapy was given for 2 months, and then it was discontinued once it proved to be ineffective.

In January 2014, the patient's severe headaches returned and her CSF pressure reached $500 \mathrm{mmH}_{2} \mathrm{O}$. Apart from a further decrease of glucose $(1.88 \mathrm{mmol} / \mathrm{L})$, her CSF analysis remained essentially unchanged. The brain MRI at this time revealed a round mass in the quadrigeminal cistern, multiple cyst signals in the Sylvian fissures, and the dilated cortical sulci in addition to the lesion in left thalamus. Overall contrast enhancement was present in the cerebral meninges, the cerebellar tentorium, the cyst walls, and the quadrigeminal cistern (Figures 2A,B). The ventricular-peritoneal shunt pressure was adjusted to $200 \mathrm{mmH}_{2} \mathrm{O}$ and stereotactic biopsy of the left thalamus was performed. However, the infiltration by a few lymphocytes was the only observation. The patient's condition deteriorated as generalized seizures became more frequent. In April 2014, she was transferred to our hospital. Upon admission, abnormal neurological findings included confusion, slow light perception, limited abduction of both eyes, grade IV of muscle strength of the right arm, general hyperactive tendon reflexes in both legs, positive Babinski's sign on the right side, and positive meningeal signs. The third brain MRI, performed at this time, showed a noncontrasted, non-cystic lesion in the left paraventricular white matter and a contrasted cystic lesion in the left basal ganglia. All the previous changes were found to be more pronounced, including the cyst-like signals in the dilated cortical sulci, the mass in the quadrigeminal cistern, and the enlarged subarachnoid space, and general meningeal enhancement in the brain 
and upper segment of the cervical spinal cord (Figures 3A-C). Magnetic resonance spectroscopy (MRS) indicated a Cho/Cr wave ratio higher than 2.5 in the left thalamus and basal ganglia area (Figure 3D), and 1.0 in the right symmetric area that appeared normal on MRI (Figure 3E). Brain biopsy of the left temporal lobe was performed and spindle-shaped tumor cells were found to be spreading into the leptomeninges and subarachnoid space, which were strongly positive for glial fibrillary acidic protein (GFAP) and S100, partly positive for OLIG2 and P53, and negative for IDH1(R132H), ARTX, and NeuN (Figure 4). Ki-67 reactivity in tumor tissue was about 5\%. According to 2016 World Health Organization (WHO), classification of tumors of the central nervous system, anaplastic astrocytoma, IDH-wildtype (WHO grade III) was confirmed.
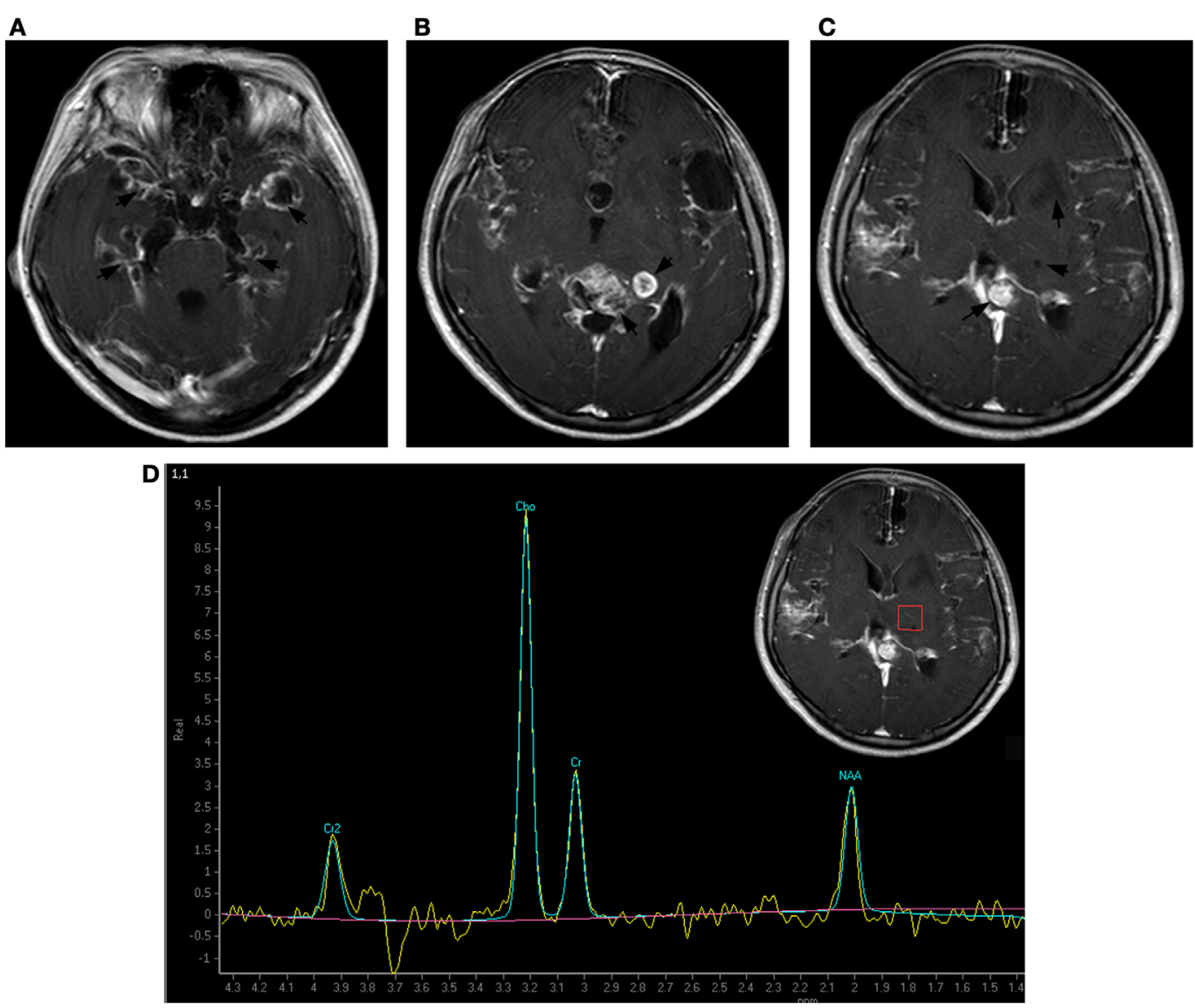

E

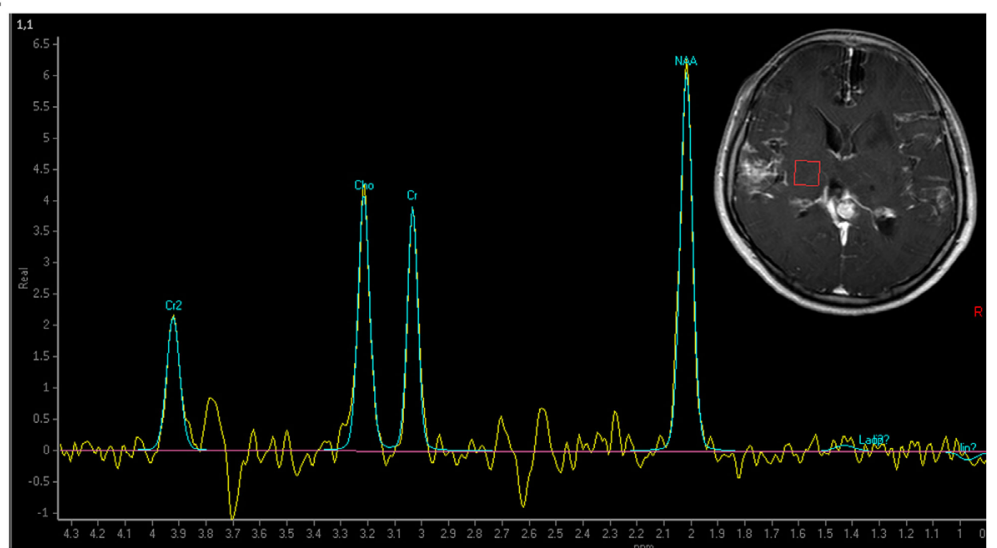

FIGURE 3 | Brain magnetic resonance imaging 1 year after symptoms onset. The subarachnoid space was markedly enlarged. More contrasted cyst-like lesions were present in the Sylvian fissure, the dilated cortex, and the left basal ganglia area and a new non-contrasted solid lesion appeared in the left paraventricular white matter. The previous left thalamus lesion was consistently unenhanced (A-C). Magnetic resonance spectroscopy indicated a Cho/Cr wave ratio higher than 2.5 in the left thalamus and basal ganglia area (D), and approximately 1.0 in the contralateral symmetric area (E). 

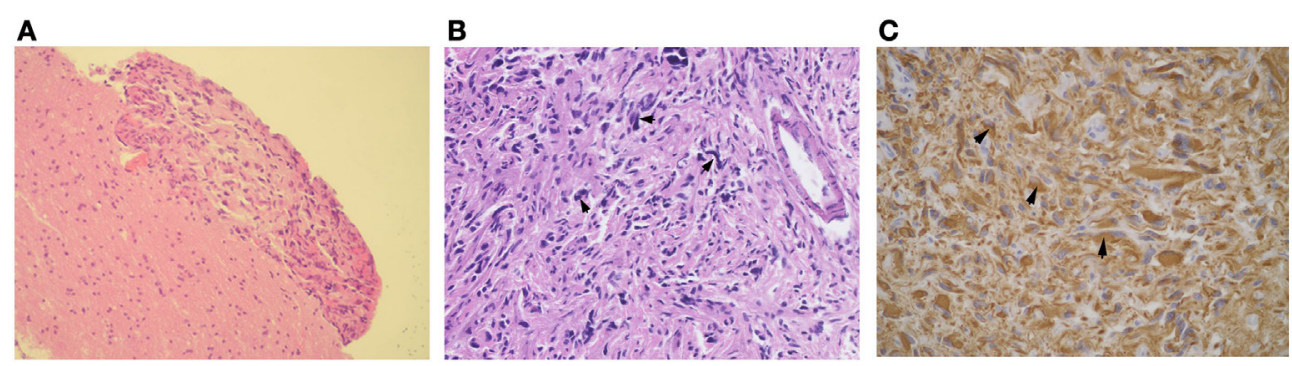

FIGURE 4 | Histological examinations of a specimen obtained in left temporal lobe. Tumor tissue was localized along the cerebral meninges (HE stain, 100x) (A). Spindle-shaped tumor cells with pleomorphic hyperchromatic nuclei and an increased mitotic rate spread in the subarachnoid space (B) (200x). The glial fibrillary acidic protein stain was strongly positive in the cytoplasm of tumor cells (C) (400x).

Combined with the extensive changes in brain MRI, PDLG was diagnosed. The patient refused treatment and was discharged from the hospital. She died 2 months later.

\section{DISCUSSION}

Primary diffuse leptomeningeal gliomatosis is a rare disease that likely arises from the heterotopic neuroglial tissue of the leptomeninges. Most PDLG patients die within the first year. Since 1951 (1), the diagnostic criteria of PDLG has been confined to the leptomeninges without involvement of the brain or the spinal cord. Until now, nearly all reported PDLG cases, except a few which described parenchyma infiltration $(2,5,6)$, were in accordance with these criteria $(7-11)$.

This patient underwent a series of brain MRI over a 10-month period. The main changes of her brain MRI are summarized as follows: (1) the progressive dilation of the subarachnoid space; (2) increasing cyst-like lesions in the Sylvian fissures, the dilated cortex sulci in the frontal lobes and the hippocampus; (3) a growing mass in the quadrigeminal cistern; (4) non-cyst-like lesions in the left thalamus and the left periventricular white matter; and (5) diffuse enhancement in the brain and the spinal meninges, the cyst walls, the subarachnoid space, and the mass in the quadrigeminal cistern.

In this case, the first MRI revealed a lesion in the dorsal of the left thalamus in addition to the dilation of the left Sylvian and diffuse enhancement of the meninges. Ten months later, the MRS revealed increased $\mathrm{Cho/Cr}$ wave ratio in the left thalamus, which is indicative of increased cellular proliferation with membrane turnover which is consistent with a neoplastic process. It is debated if the primary leptomeningeal gliomatosis infiltrated the left thalamus or if the left thalamic glioma induced secondary seeding in the leptomeninges. The solid lesion in the left thalamus was consistently non-enhanced during the 10 -month period, whereas the leptomeninges around the left thalamus was strongly nodularly enhanced even in the first MRI. A fast-growing mass was present in the quadrigeminal cistern adjacent to the left thalamus. Combined with the severe dilation, as well as the overall enhancement of the subarachnoid space and multiple encapsulated cysts in the subarachnoid space and the dilated cortical sulci, it is reasonable to speculate that the solid lesion in the left thalamus is a secondary parenchymal infiltration of PDLG in the left ventricular ependyma. The parenchyma infiltration, especially the development of non-cyst lesions in the left thalamus and the periventricular white matter, challenges the traditional diagnostic criteria of PDLG.

Our serial brain MRIs depict the pathological progression of PDLG: PDLG initiates from the leptomeninges, spreads widely in the subarachnoid space and ventricular system, then extends into cortex sulci, and at last invades the periventricular parenchyma by following the cerebral perivascular space (12). In this case, during infiltration, entrapment cysts formed in the subarachnoid space, the cortex sulci, and the left basal ganglia area. Entrapment cysts in the cortex sulci were also reported earlier by Ishige (13). The placement of ventricular drainage in our patient efficiently reduced her intracranial pressure and extended her life. This made it possible to observe the terminal multiform lesions of PDLG in the brain.

Primary diffuse leptomeningeal gliomatosis can be in the form of a solitary tumor (14) or a diffuse tumor involving intracranial or spinal cord leptomeninges $(8,15)$. Our patient was found to have a solitary tumor in the quadrigeminal cistern and diffuse leptomeningeal gliomatosis. In 2006, Kim et al. reported a case of PDLG in which they also found a mass with intensive enhancement in the quadrigeminal cistern (9).

Primary diffuse leptomeningeal gliomatosis has no special clinical and CSF features. It presents with intracranial hypertension syndromes. The increased pressure and accumulation of the exudates in the subarachnoid space may cause paralysis of multiple cranial nerves (16). CSF tests usually reveal extremely high protein level and low glucose level. The CSF routine tests can reveal normal or slightly increased white blood cells. Even though the tumor cells spread in the subarachnoid space and ventricular system, the likelihood of finding glioma cells by CSF cytology is small (8), as shown in this patient. Repeated cytology combined with immunohistochemistry may increase the diagnostic sensitivity. The overall CSF findings are consistent with tumor, infection, and sarcoidosis. In brain MRI, the hydrocephalus and overall leptomeningeal enhancement can be seen in leptomeningeal malignancy, leptomeningeal infections, and sarcoidosis. When combined with the clinical and radiological findings, leptomeningeal involvement by an infectious process or a rapidly growing neoplasm should be highly suspected. The 
dynamic alterations of PDLG as described in our patient have special characteristics that could indicate PDLG. Based on brain MRI findings, a biopsy should be done to confirm the diagnosis. The early diagnosis of PDLG will enable the early treatments of radiotherapy and chemotherapy. As reported, the treatment combining radiotherapy and temozolomide could prolong the survival time (15).

\section{CONCLUSION}

The dynamic and multiform neuroradiological changes help us to understand the pathological process of PDLG and highlight the possibility that parenchymal infiltration can occur in PDLG.

\section{ETHICS STATEMENT}

The patient's parents agreed and provided written informed consent for publication of this case report, including their child's identifiable information.

\section{REFERENCES}

1. Cooper IS, Kernohan JW. Heterotopic glial nests in the subarachnoid space; histopathologic characteristics, mode of origin and relation to meningeal gliomas. J Neuropathol Exp Neurol (1951) 10(1):16-29. doi:10.1097/ 00005072-195110010-00002

2. Riva M, Bacigaluppi S, Galli C, Citterio A, Collice M. Primary leptomeningeal gliomatosis: case report and review of the literature. Neurol Sci (2005) 26(2):129-34. doi:10.1007/s10072-005-0446-1

3. Mathews MS, Pare LS, Kuo JV, Kim RC. Primary leptomeningeal oligodendrogliomatosis. J Neurooncol (2009) 94(2):275-8. doi:10.1007/s11060-009-9821-8

4. Rodriguez FJ, Perry A, Rosenblum MK, Krawitz S, Cohen KJ, Lin D, et al. Disseminated oligodendroglial-like leptomeningeal tumor of childhood: a distinctive clinicopathologic entity. Acta Neuropathol (2012) 124:627-41. doi:10.1007/s00401-012-1037-x

5. Davila G, Duyckaerts C, Lazareth JP, Poisson M, Delattre JY. Diffuse primary leptomeningeal gliomatosis. J Neurooncol (1993) 15(1):45-9. doi:10.1007/BF01050262

6. Ashworth B, Gordon A. Leptomeningeal gliomatosis. JNeurol Neurosurg Psychiatry (1994) 57(4):471-3. doi:10.1136/jnnp.57.4.471

7. Yamasaki K, Yokogami K, Ohta H, Yamashita S, Uehara H, Sato Y, et al. A case of primary diffuse leptomeningeal gliomatosis. Brain Tumor Pathol (2014) 31(3):177-81. doi:10.1007/s10014-014-0176-2

8. Kitahara M, Katakura R, Wada T, Namiki T, Suzuki J. Diffuse form of primary leptomeningeal gliomatosis. Case report. J Neurosurg (1985) 63(2):283-7. doi:10.3171/jns.1985.63.2.0283

9. Kim SH, Jun DC, Park JS, Heo JH, Kim SM, Kim J, et al. Primary diffuse leptomeningeal gliomatosis: report of a case presenting with chronic meningitis. J Clin Neurol (2006) 2(3):202-5. doi:10.3988/jcn.2006.2.3.202

10. Gardiman MP, Fassan M, Nozza P, Orvieto E, Garre ML, Milanaccio C, et al. Diffuse leptomeningeal glioneuronal tumours: clinico-pathological follow-up. Pathologica (2012) 104(6):428-31.

\section{AUTHOR CONTRIBUTIONS}

YJ participated in the treatment of the patient, did the literature search, and drafted the manuscript. JC assisted in critical revisions of the brain MRI findings. JH participated in the treatment of the patient. AP performed the brain biopsy. JZ carefully reviewed the pathological findings. YL instructed the treatment of the patient and provided critical revisions of the manuscript for important intellectual content. All authors read and approved the final manuscript.

\section{ACKNOWLEDGMENTS}

The authors are grateful to Prof. Jingwen Jiang, Department of Neurology, Beijing Hospital for careful review of this paper. The authors also appreciate the help from Professor Cheng Sha, Department of Neurosurgery, Beijing Hospital, for his suggestions on brain biopsy and treatments. The authors thank Joshua Gregory, study director in Adlego Biomedical AB, Stockholm, Sweden, for critical review of the manuscript.

11. Guo X, Zhong D, Ma W. Primary leptomeningeal medulloblastoma: a rare case. Clin Neurol Neurosurg (2012) 114(8):1181-4. doi:10.1016/j. clineuro.2012.02.042

12. Sumi SM, Leffman H. Primary intracranial leptomeningeal glioma with persistant hypoglycorrhachia. J Neurol Neurosurg Psychiatry (1968) 31(2):190-4. doi:10.1136/jnnp.31.2.190

13. Ishige S, Iwadate Y, Ishikura H, Saeki N. Primary diffuse leptomeningeal gliomatosis followed with serial magnetic resonance images. Neuropathology (2007) 27(3):290-4. doi:10.1111/j.1440-1789.2007.00760.x

14. Ng HK, Poon WS. Primary leptomeningeal astrocytoma. Case report. J Neurosurg (1998) 88(3):586-9. doi:10.3171/jns.1998.88.3.0586

15. Hansen N, Wittig A, Hense J, Kastrup O, Gizewski ER, Van de Nes JA. Long survival of primary diffuse leptomeningeal gliomatosis following radiotherapy and temozolomide: case report and literature review. Eur J Med Res (2011) 16(9):415-9. doi:10.1186/2047-783X-16-9-415

16. Somja J, Boly M, Sadzot B, Moonen G, Deprez M. Primary diffuse leptomeningeal gliomatosis: an autopsy case and review of the literature. Acta Neurol Belg (2010) 110(4):325-33.

Conflict of Interest Statement: The authors declare that the research was conducted in the absence of any commercial or financial relationships that could be construed as a potential conflict of interest.

Copyright (c) 2017 Jiang, Chen, He, Pei, Zhang and Liu. This is an open-access article distributed under the terms of the Creative Commons Attribution License (CC BY). The use, distribution or reproduction in other forums is permitted, provided the original author(s) or licensor are credited and that the original publication in this journal is cited, in accordance with accepted academic practice. No use, distribution or reproduction is permitted which does not comply with these terms. 